\title{
On flux vacua and modularity
}

\section{Rolf Schimmrigk}

Department of Physics, Indiana University South Bend, 1700 Mishawaka Ave, South Bend, IN 46634, U.S.A.

E-mail: rschimmr@iusb.edu

ABSTRACT: Geometric modularity has recently been conjectured to be a characteristic feature of flux vacua with $W=0$. This paper provides support for the conjecture by computing motivic modular forms in a direct way for several string compactifications for which such vacua are known to exist. The analysis of some Calabi-Yau manifolds which do not admit supersymmetric flux vacua shows that the reverse of the conjecture does not hold.

Keywords: Flux compactifications, Superstring Vacua, Discrete Symmetries

ArXiv EPRINT: 2003.01056 


\section{Contents}

1 Introduction 1

2 Motivic $L$-series in weighted hypersurfaces 3

$\begin{array}{lll}3 & \text { Flux vacua with modular forms } & 6\end{array}$

$\begin{array}{ll}3.1 & \text { Flux vacua for one-parameter weighted hypersurfaces }\end{array}$

3.2 The smooth degree six hypersurface $X_{3}^{6} \in \mathbb{P}_{(1,1,1,1,2)}[6] \quad 7$

3.3 A modular rank two attractor on $X_{3}^{6} \quad 9$

3.4 Flux vacua for two-parameter hypersurfaces 9

3.4.1 The octic $X_{3}^{8 \mathrm{~B}} \in \mathbb{P}_{(1,1,2,2,2)}[8] \quad 9$

3.4.2 The degree 12 hypersurface $X_{3}^{12} \in \mathbb{P}_{(1,1,2,2,6)}[12] \quad 10$

4 On the reverse of the modularity conjecture $\quad 11$

4.1 The one-parameter octic $X_{3}^{8 \mathrm{~A}} \in \mathbb{P}_{(1,1,1,1,4)}[8] \quad 11$

4.2 The two-parameter hypersurface $X_{3}^{18} \in \mathbb{P}_{(1,1,1,6,9)}[18]$

4.3 The three-parameter hypersurface $X_{3}^{24} \in \mathbb{P}_{(1,1,2,8,12)}[24] \quad 13$

$\begin{array}{lll}5 & \text { Discussion } & 13\end{array}$

$\begin{array}{lc}\text { A Motives } & 14\end{array}$

\section{Introduction}

Modularity is a theme that arises in quite different ways in string theory. From the early days of its development modular invariance on the worldsheet has been one of the cornerstones of the foundations of the theory. Historically far removed and much older than two-dimensional conformal field theory is the notion of geometric modularity, which can be traced to Klein in the late 19th century, but which emerged as a more central part in mathematics only in the 1950s and 60s. First steps in this direction were taken in the work of Eichler, Taniyama, Shimura and Weil [1] on elliptic curves, which eventually led to the insights of Langlands [2, 3] concerning higher dimensions. The latter work was originally aimed at a nonabelian generalization of class field theory, i.e. a nonabelian generalization of the relation between Artin's Galois theoretic $L$-functions and Hecke's modular $L$-series. Today the Langlands program has absorbed Grothendieck's notion of motives and subsumes in particular the grand conjecture that all motives are automorphic. In a first approximation, motives can simply be thought of as subsectors of the cohomology of the variety.

The definition of automorphic forms is not canonical in the mathematics literature and different objects are called automorphic. One clear distinction that can be drawn is 
between modularity in the classical sense of Klein and Hecke, which views modular forms as objects that are associated to the group $\operatorname{SL}(2, \mathbb{R})$, and automorphic forms which are associated to higher rank groups. It is this latter class of objects that the Langlands conjectures are concerned with, and in the present paper the distinction between modular and automorphic forms will be made along these lines.

The notion of geometric modularity did not play a role in the first exploration of string theory compactifications in the 1980s and 1990s, perhaps because Langlands' conjectures are not very precise. They are also computationally not immediately accessible, and more importantly, a physical interpretation of the purported modular and automorphic forms was lacking. A first idea for such a physical interpretation came from the question whether geometrically induced modular forms can be related to string theoretic modular forms on the worldsheet. This was pursued in a march through the dimensions, starting with the simplest possible string compactifications of complex dimension one [4]. Extensions to higher dimensions were then constructed for K3 surfaces [5], Calabi-Yau threefolds [6, 7], as well as for Fano-type mirrors of rigid CYs [8]. Modularity in families of CY varieties was explored in [9]. Related work in this direction was done later in the context of elliptic compactifications in $[10,11]$.

Recently it was suggested that modularity might also serve as an indicator for the existence of supersymmetric flux vacua in the framework of Calabi-Yau varieties [12]. For flux compactifications there are cohomological constraints for the field $G_{3}=F_{3}-\tau H_{3}$, which are conjectured to lead to modular forms in the classical sense for flux compactifications with vanishing superpotential $W$. This was supported in [12] with computations of several points in the complex moduli space of the two-parameter octic embedded in the configuration $\mathbb{P}_{(1,1,2,2,2)}[8]$. There are other prominent Calabi-Yau configurations that are known to admit such supersymmetric flux vacua and it is of interest to test the conjecture beyond this octic.

A second issue is whether the modularity conjecture can be reversed in the way suggested in ref. [12], where the authors note that one can imagine running the conjecture in reverse to use modularity results to find new supersymmetric flux compactifications. The idea that modularity implies the existence of supersymmetric flux vacua would be very useful because modularity is expected to be a common occurrence. It is however not universal, as the example of the quintic threefold already shows. In general the Langlands conjectures only suggest the existence of automorphic forms. Hence modularity is selective in the sense that not every manifold leads to classical modular forms and it is of interest to analyze manifolds that have been shown not to admit flux vacua with $W=0$.

The outline of this paper is as follows. Section 2 contains a description of the methods used to compute the motivic $L$-series derived from weighted projective hypersurfaces. Section 3 extends the analysis of [12] to several prominent Calabi-Yau threefolds that have been considered in the literature and for which flux vacua are known to exist, adding also a remark about modular black hole attractors. Section 4 addresses the issue of the reverse of the flux vacua modularity conjecture, and section 5 contains the conclusions. An appendix contains a brief and intuitive description of the theory of motives. 


\section{Motivic $L$-series in weighted hypersurfaces}

In this section we describe a method that allows to efficiently identify rank two motives $M$ for manifolds with high dimensional cohomology groups and to compute their $L$-series $L(M, s)$. These $L$-series are used to check for modularity and read off the level of their modular groups. This method will then be applied in the remainder of the paper to several manifolds that have been of interest in the context of flux vacua.

There exist different methods that can be used to compute motivic $L$-series of weighted hypersurfaces. Most common in the mathematical literature is the $p$-adic approach, but in the following the emphasis will be on methods developed in a series of papers that were aimed at relating the resulting geometrically induced modular forms to forms on the worldsheet. This was initiated in [4] in the context of elliptic curves and extended to higher dimensions in [5-8] and to families in [9]. The advantage of this method is its directness and simplicity, allowing the computation of motivic $L$-series without computing the full cohomology with subsequent factorization. In the case of elliptic curves the motivic framework is not necessary because of the simple structure of their cohomology, but it becomes important when considering K3 surfaces and higher dimensional varieties, as discussed in the above references. In order to prepare for the physical issues discussed in this paper it is useful to briefly recall some of the structures that enter the arithmetic $L$-series.

The conceptual starting point is the zeta function $Z\left(X / \mathbb{F}_{p}, t\right)$ of Artin, Schmidt, and Weil, defined as a series expansion that collects the cardinalities $N_{r, p}(X)$ of the variety $X$ defined over finite field extensions $\mathbb{F}_{p^{r}}$ of $\mathbb{F}_{p}$ as

$$
Z\left(X / \mathbb{F}_{p}, t\right)=\exp \left(\sum_{r \geq 1} \frac{N_{r, p}(X)}{r} t^{r}\right) .
$$

Dwork's unscheduled proof [13] of the rationality of the zeta function

$$
Z\left(X / \mathbb{F}_{p}, t\right)=Q_{p}(X, t) / R_{p}(X, t)
$$

in terms of polynomials $Q_{p}, R_{p}$ gives this series (2.1) a finite form that makes it useful because it shows that a finite amount of computation determines the complete structure of $Z\left(X / \mathbb{F}_{p}, t\right)$. The detailed structure of $Q_{p}, R_{p}$ was outlined by Weil [14] and proven by Grothendieck [15] as given in terms of individual factors $\mathcal{P}_{p}^{j}(X, t)$ that are associated to the cohomology groups of the variety, with degrees given by the dimension of the $j^{\text {th }}$ group

$$
\operatorname{deg} \mathcal{P}_{p}^{j}(X, t)=\operatorname{dim} H^{j}(X) .
$$

The numerator collects the factors arising from the odd-dimensional groups, while the denominator runs through the even dimensional cohomology:

$$
Q_{p}(X, t)=\prod_{j=0}^{n-1} \mathcal{P}_{p}^{2 j+1}(X, t), \quad R_{p}(X, t)=\prod_{j=0}^{n} \mathcal{P}_{p}^{2 j}(X, t) .
$$

The full cohomology group of complex deformations is often a high-dimensional object, hence the polynomials $\mathcal{P}_{p}^{j}(X, t)$ are not very useful and neither are their completely 
factorized forms. This motivates the search for smaller building blocks, first introduced by Grothendieck as motives. There are several ways in which motives can be described. The most familiar approach perhaps is via cohomological realizations using the standard cohomology groups. An alternative formulation via the concept of correspondences provides a more geometric picture that makes contact with the mathematical goal to construct an appropriate category of these objects. In the present paper, following [6], motives are viewed as representations of the Galois group of a field associated to the variety because this approach provides the simplest and most effective approach. A more detailed intuitive description of motives can be found in the appendix.

The Galois theoretic framework of motives is based on the idea that associated to a manifold $X$ is a number field $K_{X}$ and that the Galois group $\operatorname{Gal}\left(K_{X} / \mathbb{Q}\right)$ of $K_{X}$ acts on the cohomology. In the following all Galois groups are of the type $\operatorname{Gal}(K / \mathbb{Q})$ and the reference to $\mathbb{Q}$ will be dropped for simplicity of notation. The action of this Galois group is in general reducible, leading to a decomposition of the full group into irreducible sectors. These sectors can be viewed as realizations of the motives. A detailed introduction to general motives a la Grothendieck can be found in a string physics context in ref. [6], which also contains references to the standard mathematical literature on motives. This paper also describes the concrete realization of these objects that allows to specialize the abstract categorical treatment of the mathematics literature to concrete computations in the case of weighted hypersurfaces.

In the case of the manifolds of interest here the abstract definition of motives just outlined can be made concrete. The important factor comes from the intermediate cohomology and can be written as

$$
\mathcal{P}_{p}(X, t)=\prod_{\alpha}\left(1+j_{p}(\alpha) t\right)
$$

where the $\alpha$ are rational vectors of dimension five that essentially parametrize the cohomology for certain primes $p$. More generally, the set of $\alpha$ for a weighted hypersurface of Brieskorn-Pham type of complex dimension $n$ with weights $\left(w_{0}, \ldots, w_{n+1}\right)$ and degree $d$ is given by

$$
\mathcal{A}_{n}^{d, p}:=\left\{\alpha \in \mathbb{Q}^{n+2} \mid 0<\alpha_{i}<1, r_{i}=\left(d_{i}, p-1\right), r_{i} \alpha_{i} \equiv 0(\bmod 1), \sum_{i} \alpha_{i}=0(\bmod 1)\right\},
$$

where $d_{i}=d / w_{i}$.

For a vector $\alpha$ the Jacobi sum is determined in terms of characters $\chi_{\alpha_{i}}(u)$ on finite fields $\mathbb{F}_{p}$ which are defined as

$$
\chi_{\alpha_{i}}(u)=e^{2 \pi i m \alpha_{i}}
$$

where $m$ is determined in terms of the generator $g$ of $\mathbb{F}_{p}$ as $u=g^{m}$. With these characters the Jacobi sums can be written as

$$
j_{p}(\alpha)=\frac{1}{p-1} \sum_{\substack{u_{0}+\cdots+u_{n+1}=0 \\ u_{i} \in \mathbb{F}_{p}}} \chi_{\alpha_{0}}\left(u_{0}\right) \cdots \chi_{\alpha_{n+1}}\left(u_{n+1}\right) .
$$


As noted above, the full cohomology group of complex deformations of weighted hypersurfaces is often a high-dimensional space and it is more efficient to think in terms of the arithmetic building blocks of the manifolds. The simplification that arises from the motivic structure is that given any of the vectors $\alpha$ and the Galois group $\operatorname{Gal}\left(K_{X}\right)$ of the number field $K_{X}$ associated to the manifold $X$ one can consider motives generated by the orbits $\mathcal{O}_{\alpha}$ of $\alpha$ by the action this Galois group on the vector $\alpha$. This leads to a computationally useful representation of the motive as

$$
M_{\alpha}=\operatorname{Gal}\left(K_{X}\right) \cdot \alpha .
$$

While for general varieties $X$ the field $K_{X}$ of the variety is determined by the factorization of the polynomials $\mathcal{P}_{p}$ that enter the Dwork-Grothendieck factorization of the zeta function, for weighted hypersurfaces this field is immediately determined as the cyclotomic field $\mathbb{Q}\left(\mu_{d}\right)$, where $\mu_{d}$ is the cyclic group determined by the degree $d$ of the manifold. The rank of the resulting motives is generically given by the order of the associated Galois group $\operatorname{Gal}\left(\mathbb{Q}\left(\mu_{d}\right)\right)$, which is isomorphic to $(\mathbb{Z} / d \mathbb{Z})^{\times}$, and whose order is given by Euler's totient function $\phi(d)$, which can be computed via the product formula $\phi(d)=d \prod_{p \mid d}(p-1) / p$, where the product is over all prime divisors of the degree $d$. Hence the generic rank of the motive is determined by the degree of the hypersurface.

The $L$-series of this motive $M_{\alpha}$ can then be obtained via the motivic polynomials

$$
\mathcal{P}_{p}\left(M_{\alpha}, t\right)=\prod_{\beta \in M_{\alpha}}\left(1+j_{p}(\beta) t\right)
$$

as

$$
L\left(M_{\alpha}, s\right)=\sum_{n} \frac{a_{n}\left(M_{\alpha}\right)}{n^{s}}=\prod_{p} \frac{1}{\mathcal{P}_{p}\left(M_{\alpha}, p^{-s}\right)} .
$$

With the motive $M_{\alpha}$ defined as the Galois orbit (2.9) the combination of Jacobi sums that determines the coefficients in the $L$-series are rational integers even though the Jacobi sums themselves are complex. A proof, based on the fact that the action of an element $g \in \operatorname{Gal}\left(\mathbb{Q}\left(\mu_{d}\right)\right)$ on $\alpha$ induces an action on the Jacobi sum $j_{p}(\alpha)$, can be found in [5].

A second type of $L$-series are those obtained from modular forms $f(q)$ defined relative to Hecke congruence subgroups $\Gamma_{0}(N)$ of the modular group $\operatorname{SL}(2, \mathbb{Z})$ via a tensor type transformation behavior (see e.g. [6] for a physical discussion). Associated to modular forms are $L$-series via the Mellin transform, which via the expansion $f(q)=\sum_{n} a_{n} q^{n}$ leads to the same type of series $L(f, s)=\sum_{n} a_{n} n^{-s}$. The question becomes whether these $L$-series match for some weight and level $N$.

The main interest in the earlier work cited above was to apply this construction to the holomorphic threeform $\Omega$, which leads to the concept of the $\Omega$-motive [5] (see also [6]), and to relate the resulting modular and automorphic forms to the modular structure on the worldsheet. In the present paper the focus is on the existence of rank two motives and their modularity. The focus in particular will be on Calabi-Yau varieties that have been of interest in the context of flux compactifications.

An application of the arithmetic of CYs that is aimed at the connection with the string worldsheet conformal field theory, but is independent of the modular structure, can be found in ref. [16]. 


\section{Flux vacua with modular forms}

Flux vacua with contributions from both the RR field $F_{(3)}$ and the NSNS field $H_{(3)}$ in type IIB theory lead to a superpotential $W$ that is usually written in terms of the complex axion-dilaton $\tau=C_{0}+i e^{-\varphi}$ and the field $G_{(3)}=F_{(3)}-\tau H_{(3)}$ as

$$
W=\int_{X} G_{(3)} \wedge \Omega
$$

where $\Omega$ is the holomorphic threeform. The specific form of $\tau$ adopted here is motivated by the fact that it lives on upper halfplane, $\operatorname{Im}(\tau)>0$.

The vacuum constraints for the complex deformations $z^{a}$, defined as the $\Omega$-periods via a homology basis $\left\{A_{a}, B^{b}\right\}$ and its dual, as well as the axion-dilaton $\tau$, are given by

$$
D_{\tau} W=0=D_{a} W
$$

where $D_{\tau} W \equiv \partial_{\tau} W+W \partial_{\tau} K$ and $D_{a} W=\partial_{a} W+W \partial_{a} K$, where $K=K_{\tau}+K_{\text {cs }}$ is the Kähler potential of $\tau$ and the complex deformations respectively

$$
K_{\tau}=-\ln (-i(\tau-\bar{\tau})), \quad K_{\mathrm{cs}}=-\ln \left(i \int_{X} \Omega \wedge \bar{\Omega}\right)
$$

These constraints are sometimes called F-flatness. If in addition to the criticality constraints (3.2) the superpotential vanishes as well, $W=0$, the resulting vacua are called supersymmetric. While the criticality constraints determine $G_{3}$ to be of type $H^{2,1} \oplus H^{0,3}$, the vanishing of the potential imposes the further constraint $G_{3} \in H^{2,1}[17,18]$. In depth reviews of flux compactifications can be found in [19-21].

\subsection{Flux vacua for one-parameter weighted hypersurfaces}

The class of smooth Calabi-Yau hypersurfaces in weighted projective space consists of four spaces, all of which were considered first in the context of flux vacua in [22]. It was shown there that of these four only the degree six hypersurface $X_{3}^{6} \in \mathbb{P}_{(1,1,1,1,2)}[6]$ leads to flux vacua with $W=0$, while the remaining three, given by the quintic $X_{3}^{5} \in \mathbb{P}_{4}[5]$, the octic $X_{4}^{8 \mathrm{~A}} \in \mathbb{P}_{(1,1,1,1,4)}[8]$ and the degree ten hypersurface $X_{3}^{10} \in \mathbb{P}_{(1,1,1,2,5)}[10]$, do not. Thus, while all these manifolds share the property that they have the simplest possible Kähler sector with $h^{1,1}=1$, they behave quite differently. The reason for this is to be found in the fact that the number fields $K_{X}$ associated to these manifolds have different degrees in that the smooth degree six surface leads to a quadratic extension of the rationals $\mathbb{Q}$, while the remaining spaces have fields of higher degree.

Thus, if modular motives of rank two exist for any of these three manifolds (at the relevant $\psi$ ) then it is established that modularity is not sufficient for the existence of supersymmetric flux vacua. It is therefore of interest to check whether among these manifolds there exist spaces that are modular in the sense discussed here. In the case of the quintic threefold $X_{3}^{5}$ it follows from the fact that the Galois group $\operatorname{Gal}\left(K_{X_{3}^{5}}\right)=(\mathbb{Z} / 5 \mathbb{Z})^{\times}=\{1,2,3,4\}$ has order four, in combination with the structure of $H^{2,1}$ cohomology, that there are no rank two 
motives, consistent with the modularity conjecture. Similarly, for the degree ten hypersurface the Galois group also has order four, $\operatorname{Gal}\left(K_{X_{3}^{10}}\right)=(\mathbb{Z} / 10 \mathbb{Z})^{\times}=\{1,3,7,9\}$, and it follows again from the cohomological structure that all motives are of rank four. Thus in both of these cases the manifold is at best automorphic of higher rank. A similar analysis shows that the same holds for the configurations $X_{3}^{14} \in \mathbb{P}_{(1,2,2,2,7)}[14]$ and $X_{3}^{15} \in \mathbb{P}_{(1,3,3,3,5)}[15]$, which have $\left(h^{1,1}, h^{2,1}\right)=(2,122)$ and $\left(h^{1,1}, h^{2,1}\right)=(3,75)$, respectively.

These considerations already provide additional support of the modularity conjecture and the discussion leaves among the one-parameter manifolds the degree six manifold, which does admit supersymmetric flux vacua, and the smooth octic, which does not. The first of these two will be computed presently, while the octic $X_{3}^{8 \mathrm{~A}}$ will be considered in the next section.

\subsection{The smooth degree six hypersurface $X_{3}^{6} \in \mathbb{P}_{(1,1,1,1,2)}[6]$}

An example that appeared early in the discussions of flux vacua based on the class of weighted hypersurfaces constructed in [23-25] is the degree six Brieskorn-Pham manifold in the configuration $\mathbb{P}_{(1,1,1,1,2)}[6]$, defined by

$$
X_{3}^{6}=\left\{\sum_{i=0}^{3} z_{i}^{6}+z_{4}^{3}=0\right\} \subset \mathbb{P}_{(1,1,1,1,2)} .
$$

This is a smooth hypersurface, hence it has only one Kähler form inherited from the ambient space, $h^{1,1}=1$, and the cohomology $H^{2,1}(X)$ is of monomial type, $h^{2,1}=103$. It was shown in ref. [22] that supersymmetric flux vacua exist for this manifold at the Landau-Ginzburg point, i.e. the constraints $D_{a} W=0=D_{\tau} W$ can be solved as well as $W=0$. Further discussions of flux vacua derived from this manifold can be found in a number of papers, including $[26,27]$.

In the context of a string theoretic interpretation of geometric modularity this variety was discussed briefly in [6], where the modular structure was established at the LandauGinzburg point described by the Brieskorn-Pham geometry. In ref. [6] the focus was on the $\Omega$-motive, spanned by $H^{3,0} \oplus H^{0,3}$ of this manifold, while in the context of flux vacua the cohomology sector $H^{2,1}(X) \oplus H^{1,2}(X)$ is of interest. By making the set $A_{n}^{d, p}$ defined in the previous section explicit for this hypersurface it becomes clear that modulo permutations there are twelve basic $\alpha$-vectors that lead to six different motivic $L$-series. These six $L$ functions arrange themselves into three pairs $L_{A \pm}, A=\mathrm{I}$, II, III, of series that differ only in the signs of some of their coefficients, indicating that they are character twists of each other. These motivic $L$-series can be identified with modular form $L$-series as follows. Parametrizing an independent set of motives by the $\alpha$-vectors

$$
\alpha_{A+} \in\left\{\left(\frac{1}{3}, \frac{1}{3}, \frac{1}{2}, \frac{1}{2}, \frac{1}{3}\right),\left(\frac{1}{6}, \frac{1}{3}, \frac{1}{3}, \frac{1}{2}, \frac{2}{3}\right),\left(\frac{1}{6}, \frac{1}{6}, \frac{1}{2}, \frac{1}{2}, \frac{2}{3}\right)\right\}
$$

and

$$
\alpha_{A-} \in\left\{\left(\frac{1}{3}, \frac{1}{3}, \frac{1}{3}, \frac{1}{3}, \frac{2}{3}\right),\left(\frac{1}{6}, \frac{1}{2}, \frac{1}{2}, \frac{1}{2}, \frac{1}{3}\right),\left(\frac{1}{6}, \frac{1}{6}, \frac{1}{3}, \frac{2}{3}, \frac{2}{3}\right)\right\}
$$




\begin{tabular}{|l|ccccccc|}
\hline Prime $p$ & 7 & 13 & 19 & 31 & 37 & 43 & 61 \\
\hline$a_{p}\left(M_{\mathrm{I} \pm}\right)$ & \pm 1 & +5 & \pm 7 & \pm 4 & +11 & $\mp 8$ & -1 \\
$a_{p}\left(M_{\mathrm{II} \pm}\right)$ & \pm 4 & +2 & $\mp 8$ & \pm 4 & -10 & $\mp 8$ & +14 \\
$a_{p}\left(M_{\mathrm{III} \pm}\right)$ & \pm 5 & -7 & $\mp 1$ & $\mp 4$ & -1 & \pm 8 & -13 \\
\hline
\end{tabular}

Table 1. Coefficients of the motivic $L$-series $L\left(M_{A \pm}, s+1\right)$ of $X_{3}^{6}$.

leads to the set of associated $L$-series $L\left(M_{A \pm}, s\right)$. The coefficients of these $L$-series are not directly fundamental though because each coefficient at a prime $p$ contains $p$ as a factor. It is therefore natural to shift the argument $s$ of the $L$-function in order to eliminate these $p$-factors. This shift corresponds to a twist in Hodge degree of the cohomology and has been important already in [6], as well as in the context of mirrors of rigid Calabi-Yau varieties considered in [8]. In the latter context this twist can involve higher powers of the primes $p$. A selection of the $L$-function coefficients $a_{p}$ that are obtained by dividing out these prime factors is shown in the table 1 .

A perusal of various databases, starting with the list of Cremona of weight two forms [28], as well as the more extensive list of forms by Meyer [29], allows an identification of these $L$-series as arising from modular forms $f_{A \pm}(q)=\sum_{n} a_{n} q^{n}, q=e^{2 \pi i \tau}$, in the sense that their $L$-series agree with the $L$-series of the motives

$$
L\left(M_{A \pm}, s+1\right)=L\left(f_{A \pm}, s\right)
$$

where the shift in $s$ implements the twist just discussed. Here the $f_{A+}$ are modular forms at levels $N=432,144,108$, and the $f_{A-}$ are modular forms of weight two at levels $N=$ $27,36,432$, respectively. All these forms are relative to the Hecke congruence group $\Gamma_{0}(N)$. The identification via the level $N$ is sufficient for $N=27,36$ and $N=108$, but is not unique when the space of rational forms at a given level has more than one dimension. This was taken into account by Cremona [28] by introducing further alphabetical counting labels, a strategy that was adopted in a slightly different way by the $L$-function and modular form data base (LMFDB) [30]). This is of relevance in the present discussion at the levels $N=144,432$. In the former case the modular form has the Cremona label 144A(A) and the LMFDB designation 144.2.a.a. In the latter case the two forms $f_{\mathrm{I}+}$ and $f_{\mathrm{III}-}$ both belong to the same space, but the table of coefficients shows that their $L$-series are not the same. In Cremona's list the designations are 432A and 432B, respectively, while in the LMFDB the corresponding labels are 432.2.a.e and 432.2.a.d.. The modular forms at level 27 and 144 have been considered previously in a string theoretic context in refs [4] and [33], where they were shown to admit a worldsheet interpretation for elliptic compactifications. It was explained in [33] that these forms admit complex multiplication, a symmetry that imposes a certain sparseness of the nonvanishing coefficients $a_{p}$. These forms arise because the rank two motives $M_{A \pm}$ are of complex multiplication type, determined by algebraic Hecke characters by the work of Deligne [31]. Such characters have been shown to be modular in the work of Hecke [32]. All the motives constructed in this paper are of CM type. 
The modular forms identified above can be shown to arise from the genus ten and genus four curves that are embedded in the hypersurface $X_{3}^{(6)}$ and whose $L$-function decomposes into $L$-functions of elliptic curves $E_{A}$ that lead to the modular forms $f_{A}$. Including the $L$-function of the $\Omega$-motive thus leads to a completely modular structure of the third cohomology group of the form

$$
L\left(H^{3}\left(X_{3}^{(6)}\right), s\right)=L\left(f_{\Omega}, s\right) \prod_{A \pm} L\left(f_{A \pm}, s-1\right)^{a_{A \pm}} .
$$

Here $a_{A \pm} \in \mathbb{N}$ describe the multiplicity of the motives and the modular form $f_{\Omega}$ of the $\Omega$-motive is a cusp form of weight four and level $N=108$ relative to the Hecke congruence subgroup $\Gamma_{0}(N)$, i.e. $f_{\Omega} \in S_{4}\left(\Gamma_{0}(108)\right)$.

This shows that the cohomology of the degree six threefold hypersurfaces leads to a total of seven different modular forms $f_{\Omega}, f_{A \pm}$. Combining the flux vacua analysis of [34] with the modularity analysis above thus provides further support for the flux vacua modularity conjecture of ref. [12].

\subsection{A modular rank two attractor on $X_{3}^{6}$}

A third arithmetic theme in string theory is provided by black hole attractors, initially emphasized in the number theoretic context by Moore [35], and further developed in the context of complex multiplication in refs. [36, 37]. Recently, an extensive search for rank two attractors was reported in ref. [38]. It is in this context worth noting that the modularity of the $\Omega$-motive $M_{\Omega}$ of the degree six hypersurface $X_{3}^{6}$, established in [6] and recalled briefly in the previous section, in terms of a weight four cusp form at level $N=108$, i.e. $f\left(M_{\Omega}\left(X_{3}^{6}\right), q\right) \in S_{4}\left(\Gamma_{0}(108)\right.$, shows that the rank two attractor derived from the smooth degree six hypersurface is modular in the classical sense.

The form $f\left(M_{\Omega}\left(X_{3}^{6}\right), q\right)$ of $X_{3}^{6}$ has complex multiplications, which provides a second way to recognize complex multiplication as a characteristic of at least some black hole attractors. This is independent of the approach in $[36,37]$ via the Shioda-Katsura decomposition of the cohomology of curves embedded in weighted hypersurfaces. The Deligne conjecture, discussed in the context of black hole attractors in [36], has been proven for motives with complex multiplication [39], hence leads to a relation between the periods of the variety and the $L$-functions values of $M_{\Omega}$. Since the black hole potential can be written in terms of the periods it immediately follows from the work of [36] and [6] that the entropy can be expressed in terms of these $L$-function values. This was recently made explicit in a different example in [38] and is further discussed in [40].

\subsection{Flux vacua for two-parameter hypersurfaces}

\subsubsection{The octic $X_{3}^{8 \mathrm{~B}} \in \mathbb{P}_{(1,1,2,2,2)}[8]$}

The resolved Brieskorn-Pham octic hypersurface

$$
X_{3}^{8 \mathrm{~B}}=\left\{z_{0}^{8}+z_{1}^{8}+z_{2}^{4}+z_{3}^{4}+z_{4}^{4}=0\right\} \subset \mathbb{P}_{(1,1,2,2,2)}
$$

has Hodge numbers $h^{1,1}=2$ and $h^{2,1}=86$, where both sectors receive contributions of the resolution of the singular set. This variety is a K3 fibration with a typical fiber in the 
configuration of quartic surfaces $X_{2}^{4} \in \mathbb{P}_{3}[4]$, which has been considered in the context of flux vacua in a number of papers, including $[12,26,41]$. In the latter reference this is the main configuration considered.

Aspects of a string theoretic interpretation of geometrically induced modular forms of weight two were discussed for this variety in [42], where it was shown that this manifold leads to a weight two modular form at level $N=64$. This modular form arises from the algebraic curve $C^{4}$ of degree four that defines the singular set embedded in this manifold. While this curve has genus three, its $L$-function factorizes, in the process leading to an elliptic curve of degree four, which is modular at the given level. This form was also considered in the paper by Kachru, Nally and Yang [12] as support for the modularity conjecture.

In the motivic framework considered in this paper the starting point is the Galois group $K_{X}$, which in the present example has order four

$$
\operatorname{Gal}\left(K_{X_{3}^{8 \mathrm{~B}}}\right) \cong\{1,3,5,7\} .
$$

Hence the generic orbit has length four, which is in particular the case for the $\Omega$-motive $M_{\Omega}$. Such rank four motives are not modular but are expected to be automorphic according to the Langlands conjecture. Having a Galois group whose order is larger than two in general does not prevent the existence of orbits of shorter length, leading to the possibility of rank two motives that can be modular. For the present case of the two-parameter octic modular motives of rank two can be found, for example, at levels $N=32,64$ for the motives parametrized by $\alpha=\left(\frac{1}{4}, \frac{1}{4}, \frac{1}{4}, \frac{1}{2}, \frac{3}{4}\right)$ and $\left(\frac{1}{4}, \frac{1}{4}, \frac{1}{2}, \frac{1}{2}, \frac{1}{2}\right)$, respectively, after implementing the Tate twist.

The focus here has been on the Landau-Ginzburg point, geometrically given by the Brieskorn-Pham point in the configuration. However, the observation just made, that higher order Galois groups can lead to rank two motives, generalizes to the case of deformations of the variety away from the diagonal form. This can be seen in the motivic framework by considering the theory of deformed motives in ref. [9].

\subsubsection{The degree 12 hypersurface $X_{3}^{12} \in \mathbb{P}_{(1,1,2,2,6)}[12]$}

A second two-parameter threefold that has been discussed extensively in the flux compactification context is the resolved hypersurface

$$
X_{3}^{12}=\left\{z_{0}^{12}+z_{1}^{12}+z_{2}^{6}+z_{3}^{6}+z_{4}^{2}=0\right\} \subset \mathbb{P}_{(1,1,2,2,6)},
$$

with $h^{1,1}=2$ and $h^{2,1}=128$. This is a K3 fibration with a typical smooth fiber in the configuration $X_{2}^{6} \in \mathbb{P}_{(1,1,1,3)}[6]$ that has been analyzed in refs. [26, 34, 43-45], and more recently in ref. [46].

The order of the Galois group here is $\phi(12)=4$, hence generically the motives will again be of rank four, much like in the case of the octic hypersurface considered above. Similar to the octic case there exist rank two motives for this variety as well. It turns out that most of these rank two motives have already been encountered above in the discussion of the degree six threefold $X_{3}^{6}$ even though the orders of the underlying Galois groups are 
different. As a result, their modularity follows immediately from the analysis of section 3.2. The remaining rank two motives of this manifold can either be recovered from the twoparameter model $X_{3}^{8 \mathrm{~B}}$ considered above, or can be computed separately, leading to modular forms already encountered in our previous computations.

\section{On the reverse of the modularity conjecture}

A natural question in the context of the flux vacua modularity conjecture is whether its reverse also holds, an issue that was addressed in [12]. A positive answer to this question would be very interesting because it would imply that the existence of modular motives provides a diagnostic for the existence of flux vacua $D_{\alpha} W=0=D_{\tau} W$ for which $W=0$. This can be discussed by testing the existence of modular rank two motives in compactifications for which such $W=0$ do not exist.

As noted above already, it has been known for quite some time that among the oneparameter models there are examples for which there exist no supersymmetric flux vacua, i.e. there are points in the moduli space for which the flux dynamics as well as $W=0$ cannot be solved [22]. An example of this type that was analyzed in $[22,26,34,47]$ is the smooth octic hypersurface $X_{3}^{8 A} \subset \mathbb{P}_{(1,1,1,1,4)}[8]$ at the Brieskorn-Pham point, or Landau-Ginzburg point. As noted above already, it was furthermore shown in $[22,26]$ that among the smooth weighted CY hypersurfaces there is only one manifold that admits such supersymmetric flux vacua, namely the degree six hypersurface $X_{3}^{6} \in \mathbb{P}_{(1,1,1,1,2)}[6]$, analyzed in the previous section while the remaining three do not. It was shown in the previous section that both the quintic and the degree ten hypersurface are not modular in the classical sense defined here, which leaves the smooth octic.

\subsection{The one-parameter octic $X_{3}^{8 \mathrm{~A}} \in \mathbb{P}_{(1,1,1,1,4)}[8]$}

The next space in the sequence of manifolds with $h^{1,1}=1$ is the smooth octic

$$
X_{3}^{8 A}=\left\{p\left(z_{i}\right)=\sum_{i=0}^{3} z_{i}^{8}+z_{4}^{2}=0\right\} \subset \mathbb{P}_{(1,1,1,1,4)}
$$

of Brieskorn-Pham type, with Hodge numbers $h^{1,1}=1$ and $h^{2,1}=149$. The orientifold projection is derived in [34] via Sen's method [48] from the CY fourfold $X_{4}^{24} \in \mathbb{P}_{(1,1,1,1,8,12)}$ [24], whose Euler number $\chi=23,328$ and cohomology has been computed in ref. [49]. The associated data can be found in [50], where it is the third model in the list of elliptic fibrations. In the context of flux vacua this manifold is also considered in [22, 26, 47], and more recently in [51, 52].

The Galois group of the field $K_{X}$ of this degree eight hypersuface is of order four

$$
\operatorname{Gal}\left(K_{X}\right)=(\mathbb{Z} / 8 \mathbb{Z})^{\times}=\{1,3,5,7\}
$$

hence the $\Omega$-motive of this variety is of rank four and is expected to be automorphic according to the Langlands conjectures. While the generic orbit in the intermediate cohomology thus has length four, in the context of flux vacua the interest is again in the existence of 


\begin{tabular}{|c|cccccc|}
\hline Prime $p$ & 5 & 13 & 17 & 29 & 37 & 41 \\
\hline$a_{p}\left(M_{ \pm}\right)$ & \pm 2 & $\mp 6$ & +2 & \pm 10 & \pm 2 & +10 \\
\hline
\end{tabular}

Table 2. $L$-series coefficients for the motives $M_{ \pm}$of $X_{3}^{8 \mathrm{~A}}$.

lower rank motives. In principle one can enumerate the motives as orbits, but the main issue in the present discussion is whether there exist rank two modular motives in this variety. Examples of two-dimensional Galois orbits are generated by the vectors

$$
\alpha_{+}=\left(\frac{1}{4}, \frac{1}{4}, \frac{1}{2}, \frac{1}{2}, \frac{1}{2}\right), \quad \alpha_{-}=\left(\frac{1}{4}, \frac{1}{4}, \frac{1}{4}, \frac{3}{4}, \frac{1}{2}\right)
$$

hence they parametrize rank two motives, denoted here by $M_{ \pm}$. The computation of the motivic $L$-function associated to $M_{ \pm}$along the lines of section 2 leads to rescaled coefficients $a_{p}$ as collected in table 2 .

The existing data bases again allow to identify these $L$-series as arising from weight two modular forms $f_{ \pm}(q)$

$$
L\left(f_{ \pm}, s\right)=1 \pm \frac{2}{5^{s}}-\frac{3}{9^{s}} \mp \frac{6}{13^{s}}+\frac{2}{17^{s}}-\frac{1}{25^{s}} \pm \frac{10}{29^{s}} \pm \frac{2}{37^{s}}+\frac{10}{41^{s}}+\cdots
$$

which shows that the motives $M_{ \pm}$are modular after the Tate twist

$$
L\left(M_{ \pm}, s+1\right)=L\left(f_{ \pm}, s\right) .
$$

Here the weight two modular forms $f_{ \pm}$are at levels $N=64$ and $N=32$, respectively. At these levels the spaces of rational forms are one-dimensional, hence $N$ determines these forms uniquely. These modular forms have appeared previously in the string theory analysis of [42] and [33].

The analysis here thus leads to the conclusion that the one-parameter octic BrieskornPham hypersurface embedded in $\mathbb{P}_{(1,1,1,1,4)}$ is modular in the sense of carrying rank two motives that are modular in the classical sense, while not admitting supersymmetric flux vacua.

\subsection{The two-parameter hypersurface $X_{3}^{18} \in \mathbb{P}_{(1,1,1,6,9)}[18]$}

The above analysis for $X_{3}^{8 \mathrm{~A}}$ can be applied to other weighted hypersurfaces that have been considered in the context of the existence/nonexistence of $W=0$ flux vacua. One example that was considered early on in many papers on flux vacua, and which has received recent attention, is the degree 18 hypersurface

$$
X_{3}^{18}=\left\{z_{0}^{18}+z_{1}^{18}+z_{2}^{18}+z_{3}^{6}+z_{4}^{2}=0\right\} \subset \mathbb{P}_{(1,1,1,6,9)},
$$

see e.g. [26, 45, 53-61]. This is an elliptic fibration with $h^{1,1}=2, h^{2,1}=272$, hence $\chi=$ -540 , with a typical fiber in the configuration $E^{6} \in \mathbb{P}_{(1,2,3)}[6]$. An analysis of DeWolfe [26] based on $\mathrm{R}$ symmetries first showed that there are no $W=0$ flux vacua associated to this manifold. It is therefore again of interest to consider the motivic structure of this manifold. In this case the Galois group has order six

$$
\operatorname{Gal}\left(K_{X_{3}^{18}}\right)=\{1,5,7,11,13,17\}
$$


and hence the rank of the motives will generically be six. This holds in particular for the $\Omega$-motive, which therefore is expected to be automorphic, not modular. However, despite this larger Galois group this manifold does have rank two motives.

As before, we consider the image of $\alpha$-vectors under the Galois group. An example of a rank two motive is given by

$$
M=\left(\frac{1}{3}, \frac{1}{3}, \frac{1}{2}, \frac{1}{3}, \frac{1}{2}\right) \oplus\left(\frac{2}{3}, \frac{2}{3}, \frac{1}{2}, \frac{2}{3}, \frac{1}{2}\right) .
$$

The comparison of this motive with the rank two motive $M_{\mathrm{I}+}\left(X_{3}^{6}\right)$ of the degree six hypersurface $X_{3}^{6} \in \mathbb{P}_{(1,1,1,1,2)}[6]$ considered above shows that $M$ is identical to $M_{\mathrm{I}+}$ even though the Galois groups have different orders. It follows that the $L$-series has the same expansion as in the case of the motive $M_{\mathrm{I}+}$ of the degree six hypersurface, hence leads again to the level $N=432$ modular form designated by the LMFDB as 432.2.a.e. There are further rank two motives that arise in the manifold and these can be analyzed in a similar way.

\subsection{The three-parameter hypersurface $X_{3}^{24} \in \mathbb{P}_{(1,1,2,8,12)}[24]$}

As a final example consider the variety

$$
X_{3}^{24}=\left\{z_{0}^{24}+z_{1}^{24}+z_{2}^{12}+z_{3}^{3}+z_{4}^{2}=0\right\} .
$$

The configuration is iteratively structured in that it is not only an elliptic fibration with typical fiber in the configuration $E^{6} \in \mathbb{P}_{(1,2,3)}[6]$, but also a K3-fibration with typical fiber in the configuration $X_{2}^{12} \in \mathbb{P}_{(1,1,4,6)}[12]$. Its flux vacuum structure was considered in DeWolfe [26] as a three-parameter model with Hodge numbers $h^{1,1}=3$ and $h^{2,1}=$ 243, leading to a Kähler structure that is more involved than the often considered twoparameter examples. Other work on this manifold in the context of flux compactifications includes [62, 63].

The manifold $X_{3}^{24}$ has the highest degree $d=24$ of the manifolds considered in this paper, leading to the largest Galois group of all examples. Since the totient function here is $\phi(24)=8$, a typical Galois orbit leads to a motive of rank eight. It turns out that despite the large order of this group, the modular structure of this manifold is in part similar to that of the degree six smooth hypersurface $X_{3}^{6}$ because their motivic structure partially overlaps. The modular analysis of section 3 thus immediately leads to the existence of modular rank two motives for the manifold $X_{3}^{24}$.

\section{Discussion}

In this paper the modular and automorphic structure of most of the Calabi-Yau manifolds considered in the context of flux compactifications has been established. The methods used here are direct and allow the computation of the necessary $L$-functions from the motive itself, rather than from the factorization of the full cohomology. These results have implications for the modularity conjecture for supersymmetric flux vacua formulated in [12]. 
Support for the conjecture has been provided above by establishing the existence or nonexistence of modular rank two motives for all one-parameter smooth Calabi-Yau hypersurfaces of dimension three. While the smooth degree six and eight weighted hypersurfaces admit such modular motives, the quintic and degree ten hypersurfaces do not.

A second issue is raised by the question whether rank two modularity of motives in the cohomology sector $H^{2,1}(X) \oplus H^{1,2}(X)$ can be used as an indicator for the existence of supersymmetric flux vacua. Since modularity is expected to be common this would lead to the expectation that the existence of supersymmetric flux vacua is in some sense also generic. This was first addressed here by considering the one-parameter hypersurface of degree eight in the weighted projective space $\mathbb{P}_{(1,1,1,1,4)}[8]$. For this variety modular rank two motives can be constructed, thereby establishing that modularity is not a definitive diagnostic for the existence of flux vacua with vanishing superpotential. Other manifolds that have been considered in the flux vacua literature have a similar structure. Among these are the degree 18 configuration $X_{3}^{18} \in \mathbb{P}_{(1,1,1,6,9)}[18]$ and the degree 24 configuration $X_{3}^{24} \in \mathbb{P}_{(1,1,2,8,12)}[24]$, both of which were considered in the flux context in [26], where it was shown that these two spaces do not admit flux vacua with vanishing superpotential. The first of these is a prominent two-parameter model, while the latter has a three-dimensional Kähler sector. Both of these spaces have rank two motives that are modular, similar to the one-parameter octic.

The results of this paper thus strengthen the modularity conjecture of ref. [12] but establish that modularity is not a necessary ingredient for the existence of supersymmetric flux vacua.

\section{A Motives}

This appendix provides a brief summary of the conceptual background for the motivic constructions given in the main part of this paper.

The theory of motives has its roots in the work of Hurwitz from the late 19th century and Deuring from the 1930s, but as a conceptual framework it was initiated by Grothendieck in the 1960s. In the intervening decades it has shown persistent resistance to a complete understanding. This is in particular the case for mixed motives that are important in some physical constructions [9], but do not play a role in the present paper. For the discussion here it therefore suffices to focus on the theory of pure motives because the motives that are considered in the main text are not affected by the resolution of the weighted hypersurface. Even in the case of pure motives the properties that are expected of these objects depend on conjectures formulated originally by Grothendieck that have not been proven. An extensive discussion of the geometric theory of motives can be found in the book [64], and some aspects of the arithmetic theory are discussed in ref. [65]. More extensive reviews in a physical context can be found in $[6,12]$. Most of the mathematical literature on motives is concerned with the categorical aspects of the theory. This is important for the basic philosophy of what the theory of motives is supposed to achieve, and it is also of interest for some physical applications $[5,6]$. However, for the questions addressed 
in the present paper this is not necessary. The notation here is as light as possible in order to focus on the conceptual picture.

As mentioned in the main text, there are two main approaches to motives, one geometric, the other cohomological. In the geometric approach, based on the notion of a correspondence, motives $M$ associated to a variety $X$ are defined as triplets $M=(X, \sigma, m)$, where $\sigma$ is a projector defined by an algebraic cycle $\sigma$ in $X \times X$ relative to an equivalence relation and $m$ is an integer. The concept of a projector makes sense because correspondences can be combined and with an appropriately defined composition law they form a ring. There are several equivalence relations that can be considered for algebraic cycles and relations between different pure motives depend crucially on which of these relations is considered, as well as on the integer $m$. In category language this addresses the form of the morphisms between motives $\left(X, \sigma_{X}, m\right)$ and $\left(Y, \sigma_{Y}, n\right)$. More important for this paper is that the integer $m$ determines the rescaling of the $L$-function encountered above via the Tate twist.

The arithmetic properties of such a pure motive $M$ are obtained by considering the intersection of $\sigma$ with the graph $\Gamma_{\mathrm{Fr}_{q}^{r}}$ of the $r^{\text {th }}$ iterate of the Frobenius map $\operatorname{Fr}_{q}$ which sends a point $x$ to $x^{q}$, leading to

$$
N_{r, q}(M)=\left\langle{ }^{t} \sigma \cdot \Gamma_{\mathrm{Fr}_{q}^{r}}\right\rangle .
$$

By using the Lefschetz trace formula the cardinalities can alternatively be obtained by considering the action induced by the Frobenius automorphism $\operatorname{Fr}_{q}$ and its iterates on the ( $\ell$-adic) cohomological realizations $H_{\ell}^{i}(M)$ of the motive $M$, leading to the consideration of the traces $\operatorname{tr}\left(\operatorname{Fr}_{q}^{r} \mid H_{\ell}^{i}(M)\right)$, where the induced action is denoted by the same symbol as the Frobenius map. Here $H_{\ell}(M)$ is to be viewed as a Galois representation of some dimension that in general is different from the dimension of the cohomology group $H^{i}(X)$ in any of its usual forms. This is, in brief, the outline of the intersection theoretic and cohomological approach as it applies to the arithmetic theory of motives. In order to proceed one needs to fix a field $K$ so that the Galois group of $K$ can be used to construct motives. The last step is to obtain a description of the cohomology in such a form that the action of this Galois group is easy to deal with.

For a variety $X$ the Weil conjectures, proven by Grothendieck and Deligne, lead to a natural candidate of a field $K_{X}[5,6]$. First consider the Weil-Grothendieck decomposition of the zeta function $Z\left(X / \mathbb{F}_{p}, t\right)$ into its cohomological pieces $\mathcal{P}_{p}^{i}(X, t)$ and then decompose these further $\mathcal{P}_{p}^{i}(X, t)=\prod_{j}\left(1-\gamma_{j}^{i}(p) t^{j}\right)$. The final Weil conjecture, proven by Deligne, states that the $\gamma_{j}^{i}$ are algebraic integers with a certain norm, hence they lead to an algebraic number field constructed as $K_{X}=\mathbb{Q}\left(\left\{\gamma_{j}^{i}\right\}\right)$. In the case of the Brieskorn-Pham varieties considered above the $\gamma_{j}^{i}$ are the Jacobi sums and the number field simplifies in the way discussed earlier in section 2. The second simplification that arises for the varieties considered here is that if one considers motives based on the monomial part $H_{\text {mon }}^{3}(X)$ of the complex deformation cohomology $H^{3}(X)$ then an explicit decomposition of this group can be constructed. This decomposition is parametrized by the $\alpha$-vectors considered above and the action of the field $K_{X}=\mathbb{Q}\left(\mu_{d}\right)$ on the group $H_{\text {mon }}^{3}(X)$ can be made explicit as an action on these vectors, as described in section 2 . 


\section{Acknowledgments}

It is a pleasure to thank Ralph Blumenhagen, Ilka Brunner, and Monika Lynker for discussions and correspondence. The appendix on an intuitive and brief description of motives was added at the suggestion of the referee.

Open Access. This article is distributed under the terms of the Creative Commons Attribution License (CC-BY 4.0), which permits any use, distribution and reproduction in any medium, provided the original author(s) and source are credited.

\section{References}

[1] A. Weil, Über die Bestimmung Dirichletscher Reihen durch Funktionalgleichungen, Math. Ann. 168 (1967) 149.

[2] R. Langlands, L-Functions and automorphic representations, in Proceedings of the International Congress of Mathematicians, Helsinki Finland (1978), Acad. Sci. Fennica, Helsinki Finland (1980).

[3] R. Langlands, Automorphic representations, Shimura varieties, and motives. Ein Märchen, Proc. Symp. Pure Math. 33 (1979) 205.

[4] R. Schimmrigk and S. Underwood, The Shimura-Taniyama conjecture and conformal field theory, J. Geom. Phys. 48 (2003) 169 [hep-th/0211284] [inSPIRE].

[5] R. Schimmrigk, The Langlands program and string modular K3 surfaces, Nucl. Phys. B 771 (2007) 143 [hep-th/0603234] [INSPIRE].

[6] R. Schimmrigk, Emergent spacetime from modular motives, Commun. Math. Phys. 303 (2011) 1 [arXiv: 0812.4450] [INSPIRE].

[7] R. Schimmrigk, String Automorphic Motives of nondiagonal Varieties, arXiv:1301.2583 [INSPIRE].

[8] S. Kharel, M. Lynker and R. Schimmrigk, String modular motives of mirrors of rigid Calabi-Yau varieties, Fields Inst. Commun. 54 (2008) 47 [arXiv:0908.1256] [INSPIRE].

[9] S. Kadir, M. Lynker and R. Schimmrigk, String Modular Phases in Calabi-Yau Families, J. Geom. Phys. 61 (2011) 2453 [arXiv: 1012 . 5807] [InSPIRE].

[10] S. Kondo and T. Watari, String-theory Realization of Modular Forms for Elliptic Curves with Complex Multiplication, Commun. Math. Phys. 367 (2019) 89 [arXiv:1801.07464] [INSPIRE].

[11] S. Kondo and T. Watari, Modular Parametrization as Polyakov Path Integral - Cases with CM Elliptic Curves as Target Spaces -, arXiv:1912.13294 [INSPIRE].

[12] S. Kachru, R. Nally and W. Yang, Supersymmetric Flux Compactifications and Calabi-Yau Modularity, arXiv:2001.06022 [inSPIRE].

[13] B. Dwork, On the rationality of the zeta function of an algebraic variety, Am. J. Math. 82 (1960) 631.

[14] A. Weil, Number of solutions of equations in finite fields, Bull. Am. Math. Soc. 55 (1949) 497.

[15] A. Grothendieck, Formule de Lefschetz et rationalite de fonction de L, Seminaire Bourbaki (1964/65) 9 (1966) 41. 
[16] R. Schimmrigk, Arithmetic of Calabi-Yau varieties and rational conformal field theory, J. Geom. Phys. 44 (2003) 555 [hep-th/0111226] [INSPIRE].

[17] K. Dasgupta, G. Rajesh and S. Sethi, M theory, orientifolds and G-flux, JHEP 08 (1999) 023 [hep-th/9908088] [INSPIRE].

[18] S.B. Giddings, S. Kachru and J. Polchinski, Hierarchies from fluxes in string compactifications, Phys. Rev. D 66 (2002) 106006 [hep-th/0105097] [INSPIRE].

[19] M. Graña, Flux compactifications in string theory: A Comprehensive review, Phys. Rept. 423 (2006) 91 [hep-th/0509003] [INSPIRE].

[20] J.P. Conlon, Moduli stabilization and applications in IIB string theory, Fortschr. Phys. $\mathbf{5 5}$ (2007) 287 [hep-th/0611039] [INSPIRE].

[21] E. Pajer, Phenomenological aspects of type IIB flux compactification, Fortschr. Phys. 57 (2009) 193 [INSPIRE].

[22] O. DeWolfe, A. Giryavets, S. Kachru and W. Taylor, Enumerating flux vacua with enhanced symmetries, JHEP 02 (2005) 037 [hep-th/0411061] [INSPIRE].

[23] P. Candelas, M. Lynker and R. Schimmrigk, Calabi-Yau Manifolds in Weighted P(4), Nucl. Phys. B 341 (1990) 383 [INSPIRE].

[24] A. Klemm and R. Schimmrigk, Landau-Ginzburg string vacua, Nucl. Phys. B 411 (1994) 559 [hep-th/9204060] [INSPIRE].

[25] M. Kreuzer and H. Skarke, No mirror symmetry in Landau-Ginzburg spectra!, Nucl. Phys. B 388 (1992) 113 [hep-th/9205004] [INSPIRE].

[26] O. DeWolfe, Enhanced symmetries in multiparameter flux vacua, JHEP 10 (2005) 066 [hep-th/0506245] [INSPIRE].

[27] A. Giryavets, New attractors and area codes, JHEP 03 (2006) 020 [hep-th/0511215] [INSPIRE].

[28] J.E. Cremona, Algorithms for modular elliptic curves, Cambridge University Press, Cambridge U.K. (1997).

[29] C. Meyer, Newforms of weight two for $\Gamma_{0}(N)$ with rational coefficients, (2005).

[30] LMFDB, The L-function and modular form data base, http://lmfdb.org.

[31] P. Deligne, Valeurs de fonctions L et periodes d'integrales, Proc. Symp. Pure Math. 33 (1979) 313.

[32] E. Hecke, Mathematische Werke, Vandenhoeck \& Ruprecht, Göttingen Germany (1970).

[33] R. Schimmrigk, Arithmetic spacetime geometry from string theory, Int. J. Mod. Phys. A 21 (2006) 6323 [hep-th/0510091] [INSPIRE].

[34] A. Giryavets, S. Kachru, P.K. Tripathy and S.P. Trivedi, Flux compactifications on Calabi-Yau threefolds, JHEP 04 (2004) 003 [hep-th/0312104] [INSPIRE].

[35] G.W. Moore, Arithmetic and attractors, hep-th/9807087 [INSPIRE].

[36] M. Lynker, V. Periwal and R. Schimmrigk, Complex multiplication symmetry of black hole attractors, Nucl. Phys. B 667 (2003) 484 [hep-th/0303111] [INSPIRE].

[37] M. Lynker, R. Schimmrigk and S. Stewart, Complex multiplication of exactly solvable Calabi-Yau varieties, Nucl. Phys. B $\mathbf{7 0 0}$ (2004) 463 [hep-th/0312319] [InSPIRE]. 
[38] P. Candelas, X. de la Ossa, M. Elmi and D. Van Straten, A One Parameter Family of Calabi-Yau Manifolds with Attractor Points of Rank Two, arXiv:1912.06146 [INSPIRE].

[39] D. Blasius, On the critical values of Hecke L-series, Ann. Math. 124 (1986) 23.

[40] W. Yang, Rank-2 attractors and Deligne's conjecture, arXiv:2001.07211 [INSPIRE].

[41] R. Blumenhagen et al., A Flux-Scaling Scenario for High-Scale Moduli Stabilization in String Theory, Nucl. Phys. B 897 (2015) 500 [arXiv:1503.07634] [InSPIRE].

[42] M. Lynker and R. Schimmrigk, Geometric Kac-Moody modularity, J. Geom. Phys. 56 (2006) 843 [hep-th/0410189] [INSPIRE].

[43] A. Misra and A. Nanda, Flux vacua statistics for two-parameter Calabi-Yau's, Fortsch. Phys. 53 (2005) 246 [hep-th/0407252] [INSPIRE].

[44] J.P. Conlon and F. Quevedo, On the explicit construction and statistics of Calabi-Yau flux vacua, JHEP 10 (2004) 039 [hep-th/0409215] [INSPIRE].

[45] M. Cicoli, J.P. Conlon and F. Quevedo, Systematics of String Loop Corrections in Type IIB Calabi-Yau Flux Compactifications, JHEP 01 (2008) 052 [arXiv:0708.1873] [INSPIRE].

[46] N. Cribiori, R. Kallosh, A. Linde and C. Roupec, Mass Production of IIA and IIB dS Vacua, JHEP 02 (2020) 063 [arXiv: 1912.00027] [INSPIRE].

[47] A. Giryavets, S. Kachru and P.K. Tripathy, On the taxonomy of flux vacua, JHEP 08 (2004) 002 [hep-th/0404243] [INSPIRE].

[48] A. Sen, Orientifold limit of F-theory vacua, Phys. Rev. D 55 (1997) 7345 [hep-th/9702165] [INSPIRE].

[49] M. Lynker, R. Schimmrigk and A. Wisskirchen, Landau-Ginzburg vacua of string, M-theory and F-theory at $c=12$, Nucl. Phys. B 550 (1999) 123 [hep-th/9812195] [INSPIRE].

[50] M. Lynker, R. Schimmrigk and A. Wisskirchen, Data archive for Calabi-Yau fourfolds, http://www.th.physik.uni-bonn.de/Supplements/cy.html.

[51] A. Cole and G. Shiu, Topological Data Analysis for the String Landscape, JHEP 03 (2019) 054 [arXiv: 1812.06960] [INSPIRE].

[52] A. Cole, A. Schachner and G. Shiu, Searching the Landscape of Flux Vacua with Genetic Algorithms, JHEP 11 (2019) 045 [arXiv:1907.10072] [INSPIRE].

[53] F. Denef, M.R. Douglas and B. Florea, Building a better racetrack, JHEP 06 (2004) 034 [hep-th/0404257] [INSPIRE].

[54] V. Balasubramanian, P. Berglund, J.P. Conlon and F. Quevedo, Systematics of moduli stabilisation in Calabi-Yau flux compactifications, JHEP 03 (2005) 007 [hep-th/0502058] [INSPIRE].

[55] J.P. Conlon, F. Quevedo and K. Suruliz, Large-volume flux compactifications: Moduli spectrum and D3/D7 soft supersymmetry breaking, JHEP 08 (2005) 007 [hep-th/0505076] [INSPIRE].

[56] M. Dine and Z. Sun, R symmetries in the landscape, JHEP 01 (2006) 129 [hep-th/0506246] [INSPIRE].

[57] J.J. Blanco-Pillado et al., Inflating in a better racetrack, JHEP 09 (2006) 002 [hep-th/0603129] [INSPIRE]. 
[58] S.S. AbdusSalam, J.P. Conlon, F. Quevedo and K. Suruliz, Scanning the Landscape of Flux Compactifications: Vacuum Structure and Soft Supersymmetry Breaking, JHEP 12 (2007) 036 [arXiv: 0709.0221] [INSPIRE].

[59] R. Blumenhagen, V. Braun, T.W. Grimm and T. Weigand, GUTs in Type IIB Orientifold Compactifications, Nucl. Phys. B $\mathbf{8 1 5}$ (2009) 1 [arXiv:0811.2936] [InSPIRE].

[60] J. Louis, M. Rummel, R. Valandro and A. Westphal, Building an explicit de Sitter, JHEP 10 (2012) 163 [arXiv:1208.3208] [INSPIRE].

[61] M. Demirtas, M. Kim, L. Mcallister and J. Moritz, Vacua with Small Flux Superpotential, Phys. Rev. Lett. 124 (2020) 211603 [arXiv:1912.10047] [INSPIRE].

[62] T. Eguchi and Y. Tachikawa, Distribution of flux vacua around singular points in Calabi-Yau moduli space, JHEP 01 (2006) 100 [hep-th/0510061] [INSPIRE].

[63] N.M. Paquette and T. Wrase, Comments on $M_{24}$ representations and $C Y_{3}$ geometries, JHEP 11 (2014) 155 [arXiv: 1409.1540] [INSPIRE].

[64] J.P. Murre, J. Nagal and C.A.M. Peters, Lectures on the theory of pure motives, University Lecture Series, AMS Press, New York U.S.A. (2013).

[65] Y. André, Une introduction aux motifs (Motif purs, motifs mixtes, périodes), Société Mathématique de France, Paris France (2004). 